\title{
Resveratrol-induced autophagy in human U373 glioma cells
}

\author{
MIDORI YAMAMOTO $^{1}$, SATOSHI O SUZUKI ${ }^{2}$ and MASARU HIMENO ${ }^{1}$ \\ ${ }^{1}$ Division of Pharmaceutical Cell Biology, Faculty of Pharmaceutical Sciences, Nagasaki International University, \\ Nagasaki; ${ }^{2}$ Department of Neuropathology, Neurological Institute, Graduate School of Medical Sciences, \\ Kyushu University, Fukuoka, Japan
}

Received September 14, 2009; Accepted February 26, 2010

DOI: 10.3892/ol_00000086

\begin{abstract}
Autophagy is an intracellular protein transport process leading to the degradation of organelles and long-lived proteins in eukaryotes. The down-regulation of autophagy observed in cancer cells has been associated with tumor progression. This study investigated autophagy induced by resveratrol, a natural compound, in human glioma cells. Glioma cells were exposed to resveratrol, and the cell growth and autophagic level were evaluated. Resveratrol inhibited growth and induced cell death in U373 glioma cells. When treated with resveratrol, glioma cells stably expressing GFP fused to LC3, recruited more GFP-LC3-labeled autophagosomes, and the percentage of cells with GFP-LC3-labeled autophagosomes increased. Furthermore, in resveratroltreated glioma cells, pretreatment with P38 or ERK1/2 inhibitors reduced the autophagic level, suggesting that resveratrol-induced autophagy was positively regulated by P38 and the ERK1/2 pathway. The Akt/mTOR pathway was not involved in resveratrol-induced autophagy. Our results suggest that resveratrol has an anticancer effect on glioma cells by inducing autophagy.
\end{abstract}

\section{Introduction}

The prognosis of patients with glioblastoma, the most common primary brain tumor, is very poor. Despite the combination of surgery, radiotherapy and chemotherapy, the life expectancy of patients with glioblastoma is approximately one year from diagnosis $(1,2)$. Therefore, developing a new therapeutic strategy is imperative.

Resveratrol (trans-3,4',5-trihydroxystilbene) is a natural phytoalexin present in grapes, peanuts and red wine (3). It has been reported to possess anticancer activity based on its

Correspondence to: Dr Midori Yamamoto, Division of Pharmaceutical Cell Biology, Faculty of Pharmaceutical Sciences, Nagasaki International University, 2825-7 Huis Ten Bosch Sasebo, Nagasaki 859-3298, Japan

E-mail: midori-y@niu.ac.jp

Key words: autophagy, glioma, resveratrol striking inhibition of tumor initiation, promotion and progression (4). Furthermore, resveratrol has been shown to induce apoptotic cell death in several human cancer cell lines, such as MCF7, SW480, HCE7, Seg-1, HL60 and U251 glioma cells $(5,6)$. Although resveratrol inhibits different stages of tumor growth depending on the cell type and cellular environment, the molecular mechanism of its anticancer activity is not entirely clear.

Autophagy has multiple physiological functions including protein degradation, organelle turnover and the response of cancer cells to chemotherapy. Autophagy is induced by a variety of conditions, such as nutrient starvation, hormone treatment and cellular stress. It has been reported that malignant glioma cells are very resistant to apoptosis, but they undergo autophagy in response to chemotherapeutic agents or treatment with $\gamma$-irradiation $(7,8)$. The role of autophagy in cancer therapy is controversial in terms of whether it is protective of or detrimental to cancer cells. However, the manipulation of autophagy may lead to a novel therapeutic method for treating apoptosis-resistant cancers. The regulation of autophagy by signaling pathways overlaps with the control of cell growth, proliferation, cell survival and death (9). Although it was established that Akt/mTOR and ERK1/2 are two significant signaling pathways regulating autophagy, several signaling pathways may modulate the autophagic response in cancer cells depending, among other criteria, on the cell type, the stage of cancer development and the stromal and nutritional environment (10).

The present study investigated the anticancer effect of resveratrol on U373 human glioma cells. Resveratrol was found to inhibit cell growth in a dose-dependent manner. Resveratrol-induced autophagy in glioma cells was also demonstrated. P38 and ERK1/2 inhibitors reduced the sensitivity of resveratrol-challenged glioma cells to autophagy, indicating that P38 MAPK and ERK1/2 MAPK are involved in resveratrol-induced autophagy in human glioma cells.

\section{Materials and methods}

Reagents. Resveratrol, P38 inhibitor SB203580, ERK1/2 inhibitor PD98059 and PI3K inhibitor LY294002 were purchased from Sigma (St. Louis, MO). These were dissolved in dimethyl sulfoxide (DMSO, Sigma) to produce stock solutions. 
Cell culture and cell viability assay. U373 human glioma cells were grown in Dulbecco's modified Eagle's medium (Sigma) supplemented with $10 \%$ fetal bovine serum and $1 \%$ penicillin/ streptomycin (Sigma) at $37^{\circ} \mathrm{C}$ in $5 \% \mathrm{CO}_{2}$. The sensitivity of U373 cells to resveratrol was determined in vitro by using the cell proliferation assay reagent WST-1 (Roche Applied Science, Indianapolis, IN, USA). For this purpose, $5 \times 10^{3}$ U373 cells were seeded on a flat-bottomed 96-well microtiter plate in triplicate and cultured overnight. After cells were treated with resveratrol (20-500 $\mu \mathrm{M})$ for $48 \mathrm{~h}$, they were exposed to $10 \mu \mathrm{l}$ of the WST-1 reagent for $3 \mathrm{~h}$ at $37^{\circ} \mathrm{C}$. The absorbance at $450 \mathrm{~nm}$ was measured using a Beckman Coulter microplate reader. The viability of DMSO-treated cells was considered to be $100 \%$.

Apoptosis detection assay. U373 cells were cultured on coverslips overnight and treated with $100 \mu \mathrm{M}$ resveratrol for $48 \mathrm{~h}$. The cells were stained with the terminal deoxynucleotidyl transferase-mediated dUTP nick-end labeling (TUNEL) assay using the In Situ Cell Death Detection kit, TMR red (Roche Applied Science). Two hundred cells were counted, and the percentage of TUNEL-positive cells was scored under a microscope.

Analysis of autophagy. The pEGFP-LC3 plasmid was kindly provided by Dr N. Mizushima (Tokyo Metropolitan Institute of Medical Science, Tokyo, Japan). LC3, one of the mammalian homologues of Atg8, is widely used as a specific marker of autophagosomes (11). Transfection of the plasmids into U373 human glioma cells was performed using TransFectin Lipid Reagent (BioRad Laboratories, Hercules, CA). Stable cell lines were selected in $1 \mathrm{mg} / \mathrm{ml}$ geneticin (G418), and single clones were selected in order to yield clonal cell lines. U373 cells expressing GFP-LC 3 were treated with resveratrol for $48 \mathrm{~h}$, then fixed with $4 \%$ paraformaldehyde. The number of GFP-LC3-labeled autophagosomes per cell or the number of GFP-LC3-labeled autophagosome-positive cells was counted under a confocal microscope (LSM5 Pascal, Zeiss, Germany). The counting of GFP-LC3-labeled autophagosomes was assisted by the Metamorph software (Molecular Devices, Sunnyvale, CA).

To determine the effects of Akt/mTOR, P38 and ERK1/2 on resveratrol-stimulated autophagy in glioma cells, we evaluated autophagy in cells pre-treated with or without $15 \mu \mathrm{M}$ LY294002, $10 \mu \mathrm{M}$ SB202190 and $20 \mu \mathrm{M}$ PD98059 for $3 \mathrm{~h}$. The cells were then treated with resveratrol for $48 \mathrm{~h}$, as described above.

\section{Results}

Resveratrol inhibits survival of U373 glioma cells. To examine the effect of resveratrol on cell survival, we treated U373 cells with different doses of resveratrol $(0,20,50,100,200$ and $500 \mu \mathrm{M})$. Cell viability was measured after $48 \mathrm{~h}$ by the WST-1 assay. Cell viability decreased in a dose-dependent manner (Fig. 1A). To examine whether resveratrol induces apoptosis, we performed the TUNEL assay in U373 cells following treatment with resveratrol. Treatment with $100 \mu \mathrm{M}$ resveratrol increased the ratio of TUNEL-positive cells compared with the control-treated cells (Fig. 1B).

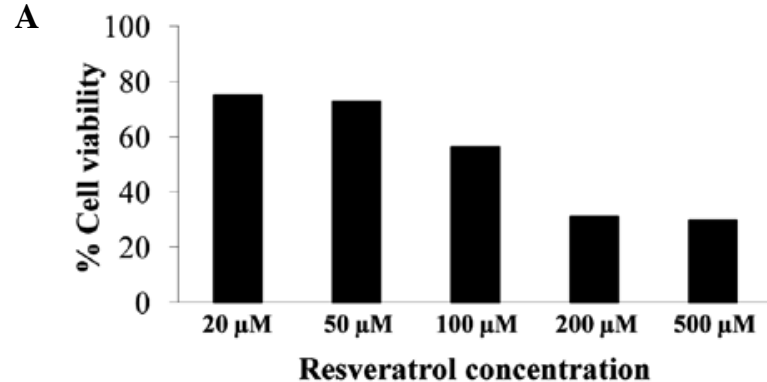

B

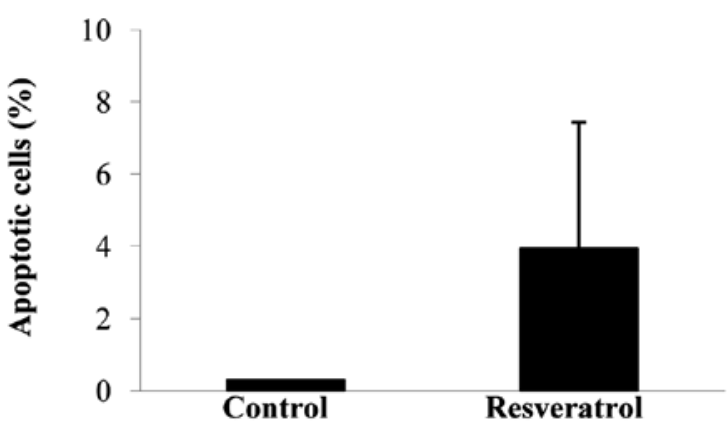

Figure 1. Effects of resveratrol on cell growth and apoptosis in glioma cells. (A) The cytotoxic effect of resveratrol as measured by the WST-1 assay. Cells were treated with different concentrations of resveratrol for $48 \mathrm{~h}$ and subjected to the WST-1 assay. The viability of DMSO-treated cells was considered to be $100 \%$. (B) Quantitation of TUNEL-positive cells. Cells were treated with $100 \mu \mathrm{M}$ resveratrol for $48 \mathrm{~h}$ and subjected to TUNEL staining. In each sample, TUNEL-positive cells were counted from among approximately 100 cells selected at random. Data are the means of triplicate experiments; error bars, SD.

Resveratrol induces autophagy in U373 glioma cells. To visualize the induction of autophagy in human glioma cells, a U373 cell line expressing GFP fused to the N-terminus of LC3 was treated with $100 \mu \mathrm{M}$ resveratrol for $48 \mathrm{~h}$. In control-treated cells, GFP-LC3 diffused throughout the cytosol (Fig. 2A). When cells were treated with resveratrol, GFP-LC3-labeled autophagosomes appeared in the cytoplasm. The percentage of autophagic cells in GFP-positive cells was $\sim 90 \%$. Furthermore, the number of GFP-positive vesicles per cell dramatically increased in cells treated with $50 \mu \mathrm{M}$ resveratrol compared with those treated with $10 \mu \mathrm{M}$ resveratrol (Fig. 2B).

P38 and ERK1/2 play a role in resveratrol-induced autophagy. It has been reported that $\mathrm{Akt} / \mathrm{mTOR}$ is the main pathway, negatively regulating autophagy, whereas the ERK pathway positively regulates autophagy in cancer cells upon starvation (9). However, the molecular mechanism regulating autophagy in response to anticancer agents remains unclear, and may vary depending on the type of cancer cell involved. To investigate the roles of PI3K, P38 and ERK1/2 kinases on resveratrol-induced autophagy in U373 cells, inhibitors of these kinases were used. As shown in Fig. 3A, the P38 SB203580 and ERK1/2 PD98059 inhibitors down-regulated the autophagic process, suggesting that both P38 and ERK1/2 promoted resveratrol-induced autophagy in glioma cells. PD98059 reduced cell viability in the resveratrol-applied system, indicating that ERK1/2 contributed to cell survival in 
A

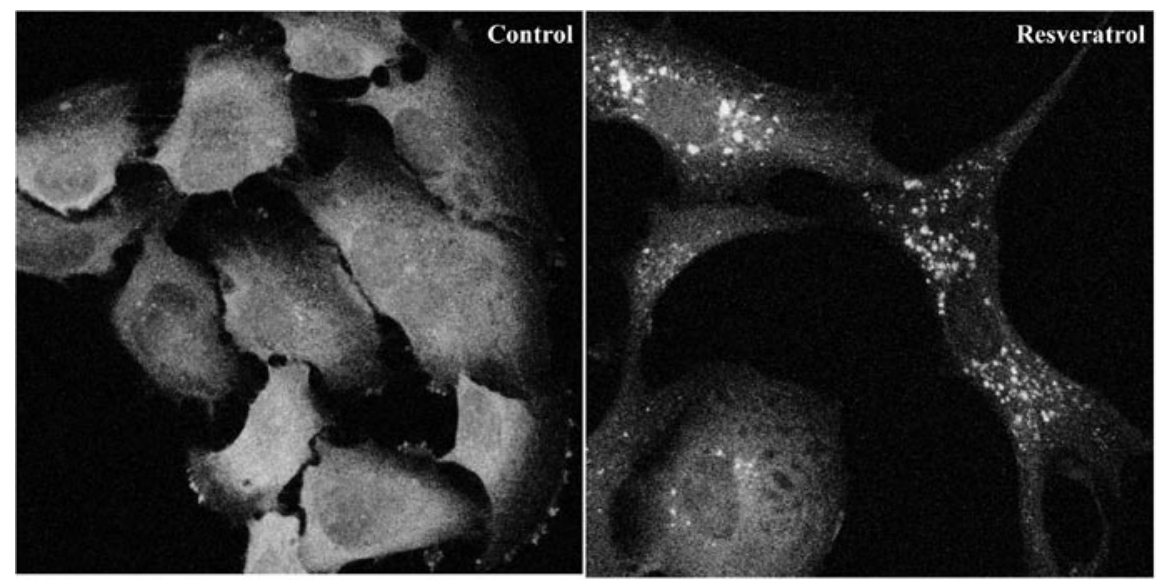

B

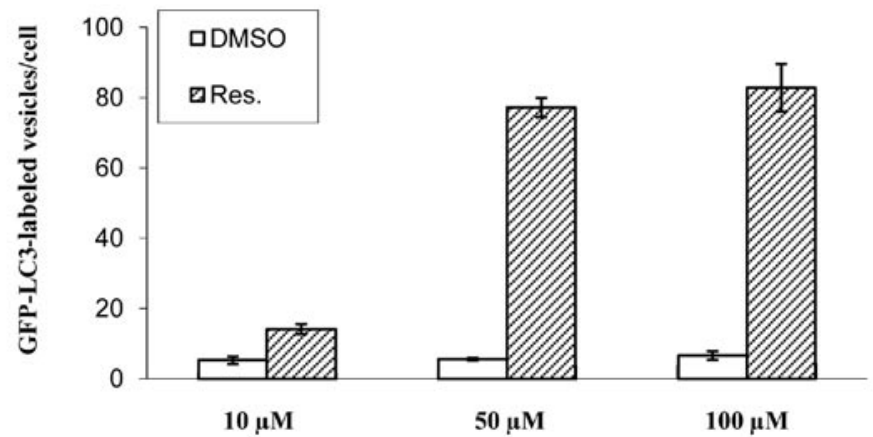

Figure 2. Resveratrol induces autophagy in glioma cells. (A) Cells expressing GFP-LC3 were cultured either with DMSO (control) (left panel) or $100 \mu \mathrm{M}$ resveratrol (right panel) for $48 \mathrm{~h}$. Magnification, x63. (B) The number of autophagosomes per cell was determined by confocal microscopy. Data are the means of triplicate experiments; error bars, SD.

this system (Fig. 3B). The PI3K inhibitor, LY294002, had no obvious effects on either resveratrol-induced autophagy nor cell viability in glioma cells (Fig. 3).

\section{Discussion}

Resveratrol, a phytoalexin found in food products such as nuts, grapes and wine, produces a variety of physiological effects. It has been reported that a low dose of resveratrol has cytoprotective activity, which is mostly attributed to its antioxidant properties. On the other hand, a high dose of resveratrol exhibits anticancer activity by interfering with different cellular events associated with the initiation, promotion and progression of multi-stage carcinogenesis $(12,13)$. The present study found that resveratrol elicited a dose-dependent inhibition of glioma cell proliferation in the micromolar range. The concentrations used were reported to result in anticancer effects in different types of cancer cells $(6,14)$.

Autophagy is a ubiquitous cellular process in eukaryotic cells that results in the breakdown of cytoplasmic organelles within the lysosomes following various types of cellular stress. This breakdown allows for the cells to respond to environmental changes or adapt to developmental processes. Opipari et al (14) reported that resveratrol inhibited cancer growth and induced autophagocytosis in ovarian carcinoma cell lines. We found that resveratrol induced autophagy in human glioma cells. Although there are multiple mechanisms by which resveratrol may exert its actions on tumor cells, autophagy may be a biological mechanism that accounts for the anticancer effects of this compound. The implication of autophagy in cancer therapy is controversial in terms of whether autophagy plays a protective or detrimental role in cancer cells. However, reduced autophagic activity has been reported in certain types of cancer cells $(15,16)$. Several natural compounds, such as arsenic trioxide, soybean B-group triterpenoid saponins or curcumin, have been shown to induce autophagy in a variety of tumor cells (17-20). Thus, compounds that induce autophagy may be of potential use as anticancer agents. Previous studies have shown that resveratrol induces apoptosis in a variety of cancer cells including glioma cells $(5,6)$. In this study, we also observed a certain degree of resveratrol-induced apoptosis in U373 cells. Autophagy and apoptosis may be triggered by common upstream signals occasionally resulting in combined autophagy and apoptosis. In other instances, however, the cell switches between the two responses in a mutually exclusive manner (21). Further research is necessary to identify the functional relationship between autophagy and apoptosis in response to resveratrol.

In mammalian cells, several signaling pathways are known to regulate autophagy in a cell type-specific and signal-dependent manner, including the ERK, PI3K class I and II, and mTOR pathways. Cancer often occurs following the deregulation of these signaling pathways. Therefore, it is important to investigate the interplay of their role in 
A
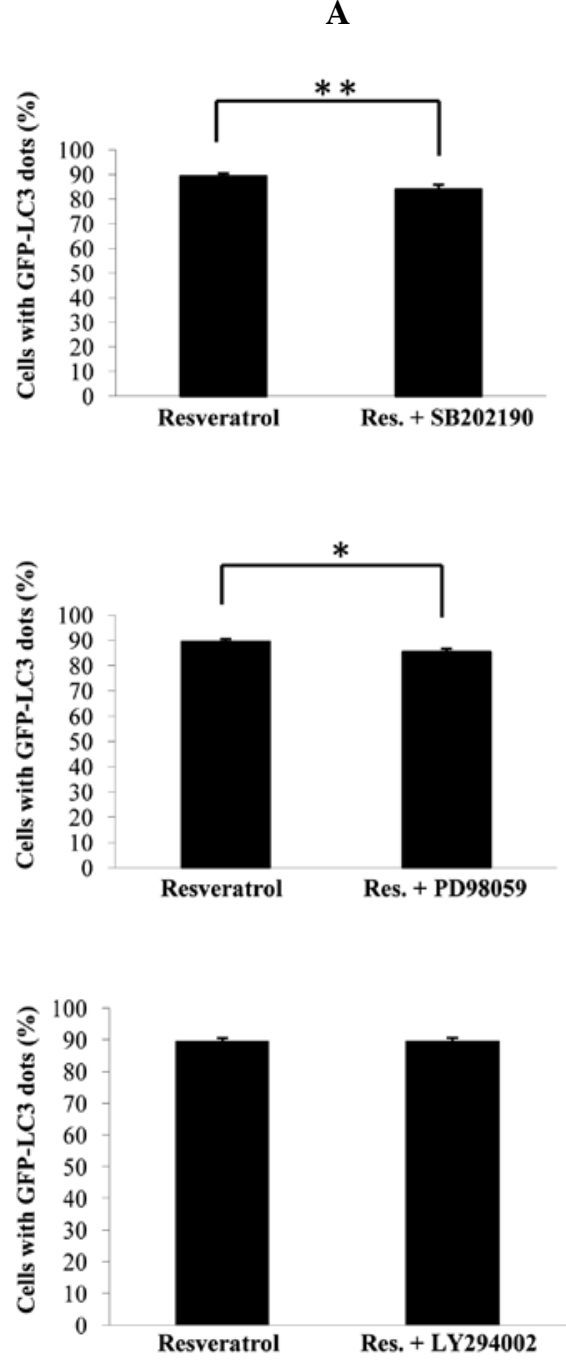
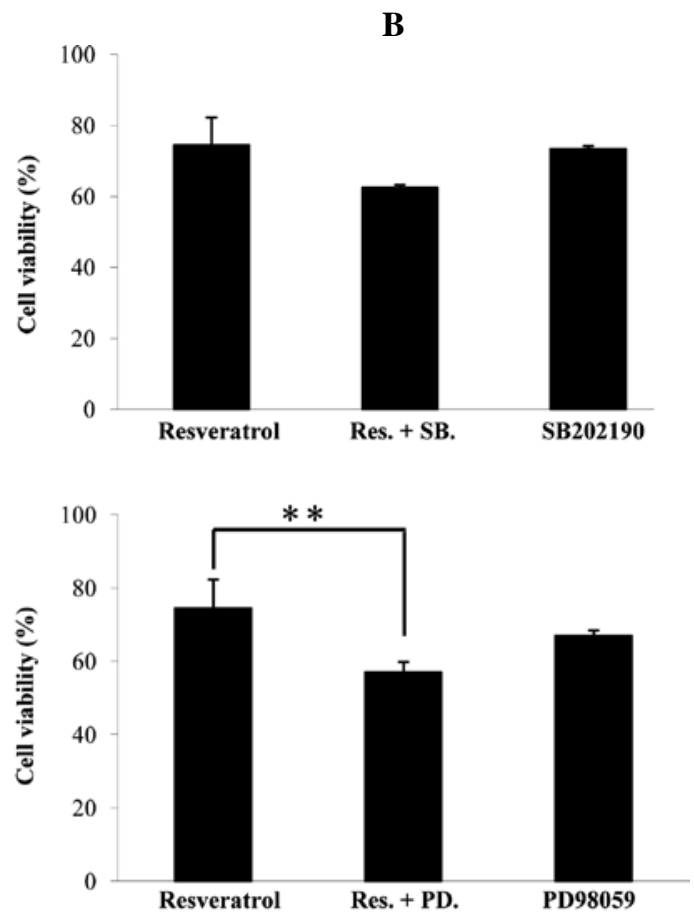

100

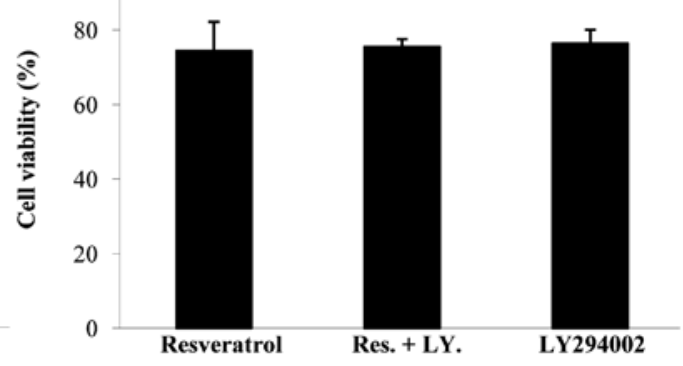

Figure 3. Effects of P38, ERK and Akt on cell autophagy and cell growth. (A) Effects of SB202190, PD98059 and LY294002 on resveratrol-induced autophagy in glioma cells. Cells were pre-incubated with these inhibitors followed by treatment with $100 \mu \mathrm{M}$ resveratrol. The number of cells with GFP-LC3-positive autophagosomes was counted, and the percentage among the total number of cells expressing GFP was determined. Data are the means of triplicate experiments; error bars, SD. (B) Effects of SB202190, PD98059 and LY294002 on the viability of resveratrol-treated glioma cells. Cells were pre-incubated with these inhibitors followed by treatment with $100 \mu \mathrm{M}$ resveratrol. Cell viability was determined by the WST-1 assay. Data are the means of triplicate experiments; error bars, SD. ${ }^{*} \mathrm{P}<0.05$; ${ }^{* *} \mathrm{P}<0.01$.

modulating autophagy, cell survival and cell growth. This study showed that resveratrol activated P38 and ERK, but not Akt/mTOR signaling, resulting in the induction of autophagy and the reduction of cell viability. Regarding the function of P38 MAPK in autophagy, abrogation of P38 by chemical inhibitors has been reported to be sufficient to interfere with the normal autophagic maturation step $(22,23)$. Our study showed that P38 was a contributing factor to resveratrol-induced autophagy. Oridonin, a herbal ent-kaurane diterpenoid, has been shown to induce autophagy by activating P38 signaling in HeLa cells, in accordance with our findings (24). The ERK and Akt pathways are known to regulate autophagy, but with opposite effects in that the ERK pathway regulates autophagy positively, whereas the Akt pathway regulates autophagy negatively (9). The natural products triterpenoid B-group soysaponins and curcumin have been shown to induce autophagy by inhibiting Akt signaling and enhancing ERK activity in human colon cancer and human glioma cells, respectively $(18,19)$. Our data showed that the inhibition of the
ERK pathway using PD98059 inhibited resveratrol-induced autophagy, but the inhibition of the Akt pathway had no effect. Cui et al (24) reported that ERK had no obvious role in oridonin-induced autophagy in HeLa cells. Further studies are needed to determine whether the combination of Akt inhibition and ERK activation is essential for the autophagic process induced by different anticancer agents in different cancer cell types.

In conclusion, our study showed that resveratrol induces autophagy and that the ERK and P38 pathways are involved in resveratrol-induced autophagy. This may offer novel therapeutic options to explore the relationship between autophagy, cell death/growth and response to anticancer agents in glioma cells.

\section{Acknowledgements}

We would like to thank Dr Noboru Mizushima for providing the pEGFP-LC3 plasmid. 


\section{References}

1. Mashaley MS Jr, Mettlin C, Natarajan N and Laws ER Jr: National survey on patterns of care for brain-tumor patients. J Neurosurg 71: 826-836, 1989.

2. Ohgaki H, Dessen P, Jourde B, et al: Genetic pathways to glioblastoma: a population-based study. Cancer Res 64: 6892-6899, 2004.

3. Gusman J, Malonne $\mathrm{H}$ and Atassi G: A reappraisal of the potential chemopreventive and chemotherapeutic properties of resveratrol. Carcinogenesis 22: 1111-1117, 2001.

4. Yang CS, Landau JM, Huang MT and Newmark HL: Inhibition of carcinogenesis by dietary polyphenolic compounds. Annu Rev Nutr 21: 381-406, 2001.

5. Loe AK, Liu H, Suzui M, Vural ME, Xiao D and Weinstein IB: Resveratrol induces growth inhibition, S-phase arrest, apoptosis and changes in biomarker expression in several human cancer cell lines. Clin Cancer Res 8: 893-903, 2002.

6. Jiang H, Zhang L, Kuo J, et al: Resveratrol-induced apoptotic death in human U251 glioma cells. Mol Cancer Ther 4: 554-561, 2005.

7. Kanazawa T, Germano IM, Komata T, Ito H, Kondo $\mathrm{Y}$ and Kondo S: Role of autophagy in temozolomide-induced cytotoxicity for malignant glioma cells. Cell Death Diff 11: 448-457, 2004.

8. Santana P, Pena LA, Haimovitz-Friedman A, Martin S McLoughlin M, Cordon-Cardo C, Schuchman EH, Fuks Z and Kolesnick R: Acid sphingomyelinase-deficient human lymphoblasts and mice are defective in radiation-induced apoptosis. Cell 86: 189-199, 1996.

9. Botti J, Djavaheri-Mergny M, Pilatte $\mathrm{Y}$ and Codogno P: Autophagy signaling and the cog wheels of cancer. Autophagy 2 : 67-73, 2006.

10. Meijer AJ and Codogno P: Regulation and role of autophagy in mammalian cells. Int J Biochem Cell Biol 36: 2445-2462, 2004.

11. Kabeya Y, Mizushima N, Ueno T, Yamamoto A, Kirisako T, Noda T, Kominami E, Ohsumi Y and Yoshimori T: LC3, a mammalian homologue of yeast Atg8p, is localized in autophagosome membranes after processing. EMBO J 19: 5720-5728, 2000.

12. Fauconneau B, Waffo-Teguo P, Huguet F, Barrier L, Decendit A and Merillon JM: Comparative study of radical scavenger and anti-oxidant properties of phenolic compounds from Vitis vinifera cell cultures using in vitro test. Life Sci 61: 2103-2110, 1997.
13. Jang M, Cai L, Udeani GO, Slowing KV, Thomas $\mathrm{CF}$, Beecher CW, Fong HH, Farnsworth NR, Kinghorn AD, Mehta RG, Moon RC and Pezzuto JM: Cancer chemopreventive activity of resveratrol, a natural product derived from grapes. Science 275: 218-220, 1997.

14. Opipari AW, Tan L, Boitano AE, Sorenson DR, Aurora A and Liu JR: Resveratrol-induced autophagy in ovarian cancer cells. Cancer Res 64: 696-703, 2004.

15. Knecht E, Hernandez-Yago J and Grisolia S: Regulation of lysosomal autophagy in transformed and nontransformed mouse fibroblasts under several growth conditions. Exp Cell Res 154: 224-232, 1984.

16. Schwarze PE and Seglen PO: Reduced autophagic activity, improved protein balance and enhanced in vitro survival of hepatocytes isolated from carcinogen-treated rats. Exp Cell Res 157: 15-28, 1985.

17. Kanazawa T, Kondo Y, Ito H, Kondo S and Germano I: Induction of autophagic cell death in malignant glioma cells by arsenic trioxide. Cancer Res 63: 2103-2108, 2003.

18. Ellington AA, Berhow $\mathrm{M}$ and Singletary KW: Induction of macroautophagy in human colon cancer cells by soybean B-group triterpenoid saponins. Carcinogenesis 26: 159-167, 2005.

19. Aoki H, Takada Y, Kondo S, Sawaya R, Aggarwal BB and Kondo Y: Evidence that curcumin suppresses the growth of malignant gliomas in vitro and in vivo through induction of autophagy: role of Akt and extracellular signal-regulated kinase signaling pathways. Mol Pharmacol 72: 29-39, 2007.

20. Rubinsztein D, Gestwicki JE, Murphy LO and Klionsky DJ: Potential therapeutic applications of autophagy. Nat Rev Drug Discov 6: 304-312, 2007.

21. Maiuri MC, Zalckvar E, Kimchi A and Kroemer G: Self-eating and self-killing: crosstalk between autophagy and apoptosis. Mol Cell Biol 8: 741-752, 2007.

22. Corcelle E, Djerbi N, Mari M, Nebout M, Fiorini C, Fenichel P, Hofman P, Poujeol P and Mograbi B: Control of the autophagy maturation step by the MAPK ERK and P38: lessons from environmental carcinogens. Autophagy 3: 57-59, 2007.

23. Simone C: Signal-dependent control of autophagy and cell death in colorectal cancer cell. Autophagy 3: 468-471, 2007.

24. Cui Q, Tashiro S, Onodera S, Minami, M and Ikejima T: Oridonin induced autophagy in human cervical carcinoma HeLa cells through Ras, JNK and P38 regulation. J Pharmacol Sci 105: 317-325, 2007. 